Itinéraires Itinéraires

Littérature, textes, cultures

\title{
Discours de gangs afro-américains sur Internet
}

Dialectique du monologue de présentation personnelle

\section{Laura Gabrielle Goudet}

\section{(2) OpenEdition}

Journals

Édition électronique

URL : http://journals.openedition.org/itineraires/2325

DOI : $10.4000 /$ itineraires.2325

ISSN : 2427-920X

Éditeur

Pléiade

Référence électronique

Laura Gabrielle Goudet, «Discours de gangs afro-américains sur Internet », Itinéraires [En ligne],

2014-1 | 2015, mis en ligne le 05 février 2015, consulté le 01 mai 2019. URL : http://

journals.openedition.org/itineraires/2325 ; DOI : 10.4000/itineraires.2325

Ce document a été généré automatiquement le 1 mai 2019.

\section{(c)}

Itinéraires est mis à disposition selon les termes de la licence Creative Commons Attribution - Pas

d'Utilisation Commerciale - Pas de Modification 4.0 International. 


\title{
Discours de gangs afro-américains sur
} Internet

\author{
Dialectique du monologue de présentation personnelle
}

\author{
Laura Gabrielle Goudet
}

\section{NOTE DE L'AUTEUR}

Je remercie vivement Marie-Anne Paveau, catalysatrice de cet article, ainsi que Nicolas Ballier et Anne Talbot pour leurs relectures patientes et éclairées. Défauts et insuffisances me restent imputables.

\section{Introduction}

1 L'identité numérique est une étape primordiale pour interagir avec d'autres utilisateurs de communautés virtuelles. Le pseudonyme en est le fer de lance, et est utilisé pour que l'utilisateur ne soit pas traçable hors ligne. Le profil ou page personnelle est la seconde étape d'identification au sein d'une communauté virtuelle, et sa fonction est de résumer la personnalité de son auteur. Dans cet article, j'examine les profils de membres de Black Planet (BP) ${ }^{1}$, le plus grand site de niche dédié aux Afro-Américains (plus de trente millions de membres) qui réunit site de rencontre, actualités, réseau social et professionnel. Ici, je n'étudie que ceux qui sont autoproclamés membres de gangs, dans une perspective discursiviste, afin d'explorer l'opposition dénotatif/connotatif, ainsi que la dialectique privé/public.

2 Le matériel apporté à chaque profil dépend des utilisateurs, et rassemble « des photos, des écrits, des vidéos, des citations dans de vastes herbiers numériques qui ont vocation [...] à refléter tout ou partie de la personnalité, des intérêts, des activités, de la mémoire » (Deseilligny 2012 : 96). En l'occurrence, cette convergence de données sert à constituer un portrait positif et idéalisé du membre de gang autoproclamé, et donc, de la culture à 
laquelle il appartient. La description personnelle revêt une forme "monologale dialogique » (Deseilligny 2012) car seul l'auteur dispose du skeptron discursif, et elle fait partie intégrante du faisceau d'information qui constitue l'identité numérique (Windley 2005).

Dans cet article, j'examine l'identité, soit la « constitution plus ou moins maîtrisée d'une image, d'une réputation» (Coutant et Stenger 2014: 35), à travers l'utilisation de technologies de soi, et d'outils numériques, ancrée dans le contexte culturel du gang afroaméricain. Les éléments culturels, identitaires en et hors ligne se complètent et offrent un tableau complet des membres. Ils suivent les exigences matérielles de BP, comme les mises en forme permises par le site (couleurs, polices, types de bannières défilantes, etc.). Je propose une analyse multimodale des effets sémiotiques produits par l'enchevêtrement des technologies utilisées. Les utilisateurs peuvent montrer leur appartenance à un groupe sur BP ou insérer des fichiers audio associés aux profils. D'autres sites peuvent être mis à contribution (via les hyperliens) et ancrer l'utilisateur de forum dans un contexte internet complexe dépassant le cadre de BP.

Le corpus a été constitué en restreignant une recherche Google à «site: www.blackplanet.com crip blood», soit les noms des deux principaux gangs à dominance afro-américaine des États-Unis. Apparus dans les années 1970 en Californie, les sousgroupes (sets) réunis sous ces étiquettes attirent l'attention des médias depuis les années 1990 (Smitherman 2000). Le corpus comprend 55 profils, dont 67,25\% de Crips et 32,75\% de Bloods ${ }^{2}$. Afin de respecter l'anonymat de ces membres, les pseudonymes cités sont écrits différemment de leur graphie sur le site. Un hypothétique «DaMu*_*66 " serait donc écrit «Damu $66^{3}$ ». Dans un premier temps, je m'intéresse à la spécificité des discours dans les profils de BP.

\section{Dynamique de la multimodalité}

5 Le profil est un collage multimodal, entre agencements textuels, chromatiques et dynamiques, dont on ne peut s'emparer comme un texte « conventionnel». Cette section analyse l'intersémioticité des différents régimes à l'œuvre. Les composantes picturales du profil et les parasitages ou le conflit de l'image fixe et de l'image en mouvement, souvent générée par des défilements de textes, le placent dans un écosystème numérique inédit (Paveau 2012), plus complexe que les mises en forme de la partie forum ou discussion instantanée de BP.

\subsection{L'espace mouvant du profil personnel}

Dans la fig. 1, le pseudonyme de Pimp Player défile sur l'image, sans jamais être entièrement lisible, des deux côtés de l'écran. Cela capte l'attention du lecteur, tout en constituant une dialectique de caché-montré : 
Fig. 1. Photographie de Pimp Player, à deux états de défilement du texte

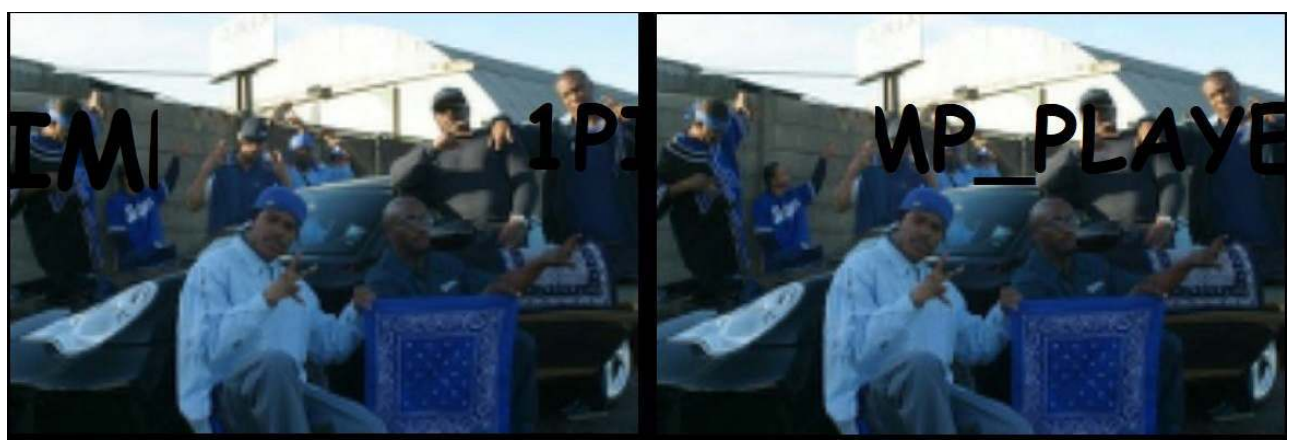

$7 \quad$ Les photographies ne sont pas des .gif, mais des .jpg ${ }^{4}$ : l'utilisateur a donc fait défiler deux zones de texte qui se superposent à l'image, et créent un discours plus stylisé que si les deux bannières étaient intégrées à l'image. Elles en débordent et créent un espace hybride texte-image, qui prend aussi le fond de la page comme support : étant donné que la page est noire, le texte n'apparait que sur la photographie.

8 Ces apparitions de texte peuvent aussi s'étaler sur toute la longueur de la page : le profil de Hood Banga s'ouvre sur une photographie, son pseudonyme y défile de droite à gauche et de gauche à droite. Sur toute la largeur de la page, le mot BOTTOMS ${ }^{5}$, dupliqué une trentaine de fois, défile de haut en bas.

Fig. 2. Défilement vertical et horizontal sur le profil de Hood Banga

BOTTOMS

BOTTOMS
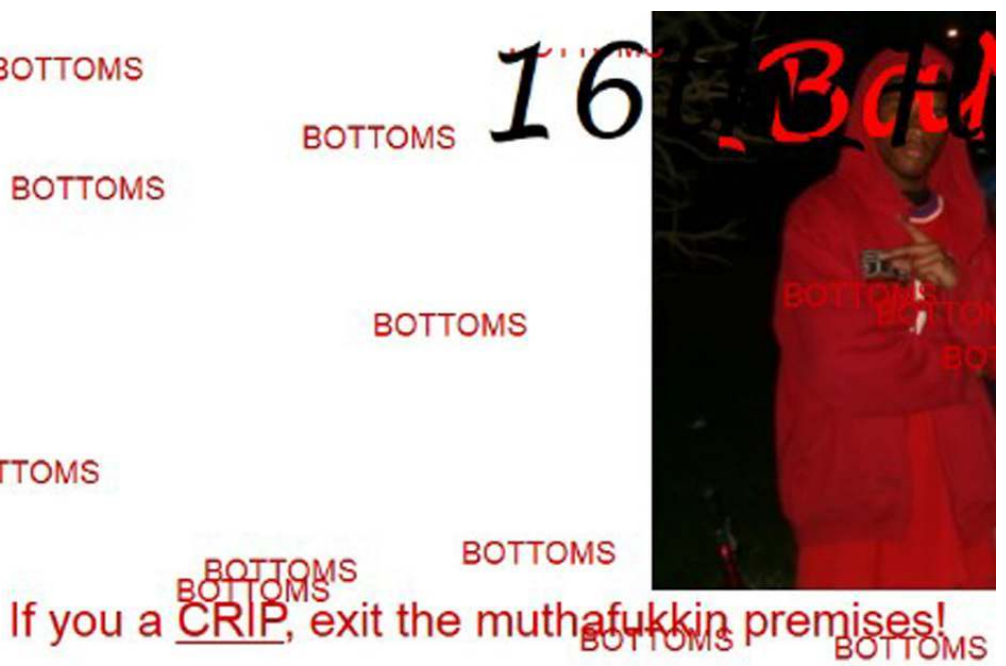

What's the deal BRBPtess take the time out and read my page befort BOTTOMS

This ya boi Lonzo coming at cha reppin those Noth"'Vkjiggs". If you an ni\&\&as don't want it on bloods ya'll pu\$\$y a\$\$ ni\&\&as about shooting v in the bottoms where it's Flat I'm out blood!

fu*k crip on bloods

Les superpositions mouvantes montrent la maitrise des outils les permettant, comme la saisie de commandes HTML, ou la prédiction intuitive de ce qu'elles créeront à l'écran. Ce qui est mobile attire l'attention du lecteur, et montre la littératie des auteurs. Sur le corpus étudié, $33 \%$ des profils sont dynamiques, les deux autres tiers ne comprennent 
que du texte ou des .gif animés, et certains (7\%) contiennent des plugins musicaux (déclenchant la musique à l'ouverture de la page). Prouver sa maîtrise d'outils qui permettent de créer une page se démarquant des autres, fait partie de l'«identité agissante» (George 2008): l'internaute témoigne de sa maîtrise du Web 2.0. Le mouvement crée une nouvelle façon de lire le profil, en faisant abstraction d'une couche textuelle pour en lire une autre, le tout au détriment de la lisibilité.

\subsection{Parasitage du discours}

10 Ces stylisations vont jusqu'à l'illisibilité du profil : la couleur de la police et le fond (couleur unie, photographie, etc.) peuvent se rendre mutuellement illisibles, comme c'est le cas dans la fig. 3.

Fig. 3. Informations personnelles de Block Boy, illisibles sans surlignement

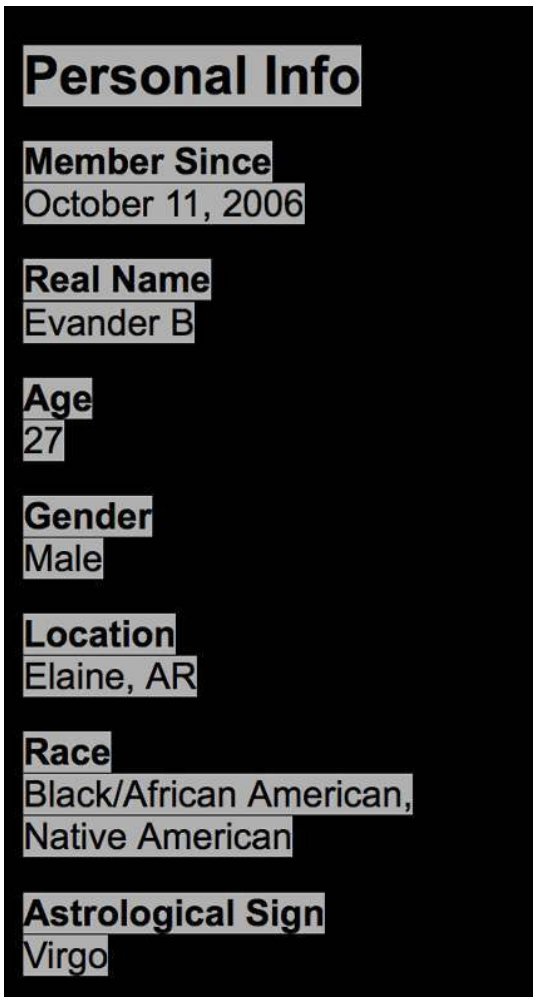

11 La sélection de couleurs et les rapports entre zones écrites et non écrites (cadres, illustrations) délimitent les éléments du profil et en permettent la séparation. Il comprend une partie didactique, sur la signification des chiffres en argot de gang (zone bleue); une description des qualités de Block Boy (en blanc); enfin, une description du partenaire qu'il cherche (en noir) dans la fig. 4. Ce profil présente plusieurs problèmes visuels, car les zones de texte se chevauchent et le palimpseste est illisible. Ceci peut être dû à l'utilisation d'illustrations permettant d'aérer la page avant la péremption de leur lien. Le cadre jaune en bas est vide, mais un texte écrit en noir apparaît lorsque la zone est mise en surbrillance. La structure de BP permet la non-linéarité textuelle et l'accumulation verticale d'éléments constitutifs. 
Fig. 4. Profil de Block Boy (1)

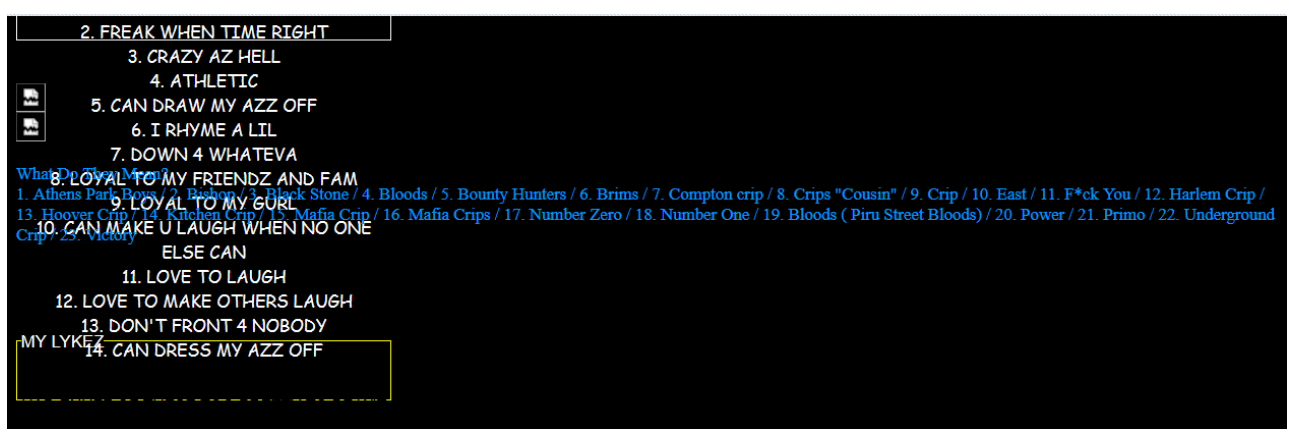

12 L'exemple de la fig. 5 fait appel à d'autres éléments textuels et visuels, dus à BP et à Block Boy. Trois types de textes se superposent. Le texte en bleu est une explication de termes afférents aux Crips, le texte en blanc est une autre explication sur les acronymes ( ARM: Aryan Resistance Militia», "MRS: Milice de Résistance Aryenne»), et le texte en noir (marqué par les boutons "Show all ») est une liste des membres amis de Block Boy. Les points d'interrogation en rouge sont des hyperliens qui renvoient à la structure de $\mathrm{BP}^{6}$, prenant ancrage dans le profil, puis dans la page d'aide du site.

13 La superposition concerne d'autres éléments: outre les strates textuelles, les icônes apparaissent sous le texte, mais les illustrations se placent en premier plan. Les deux illustrations de la fig. 5 n'ont pas de points communs : la première est un lien hypertexte vers un autre membre de BP, Syndicate, et la seconde (coupée) est une photographie de 2Pac Shakur ${ }^{7}$.

Fig. 5. Profil de Block Boy (2)

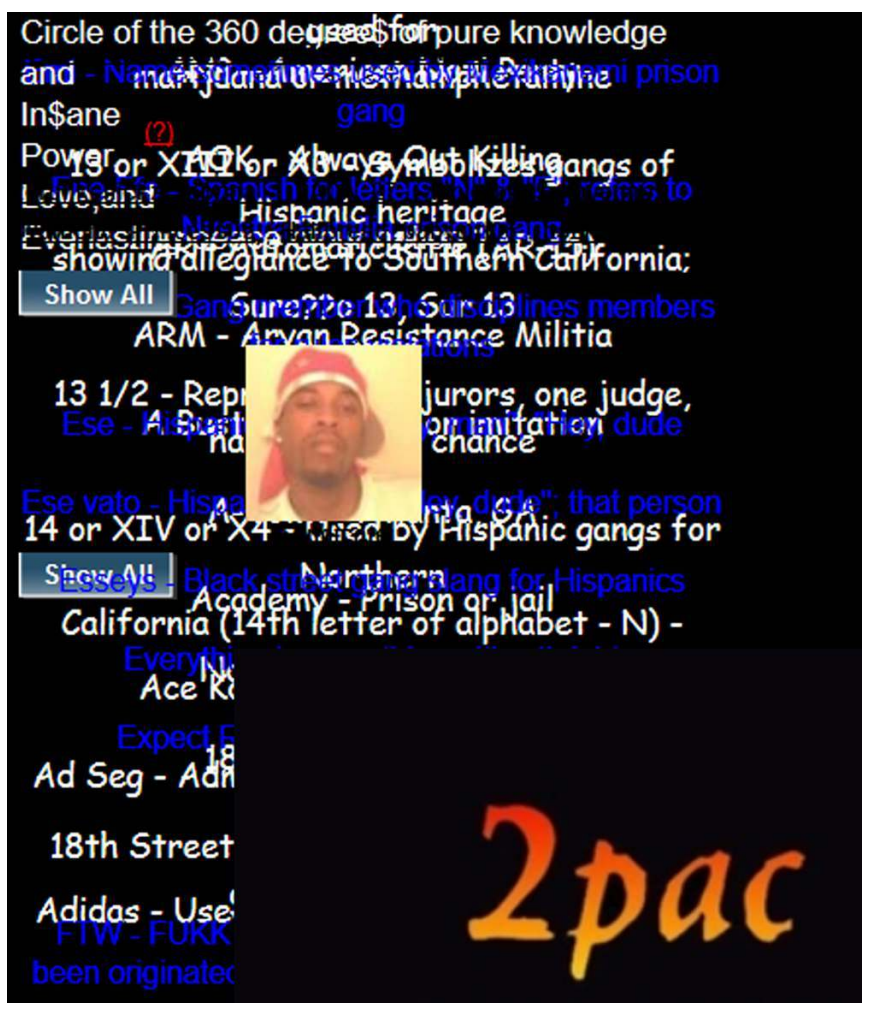


de BP. Les structures non rafraîchies des pages, dues aux auteurs ou au site, ne ressemblent à aucun autre contenu écrit sur BP (forums, chats, etc.). C'est une cacophonie numérique inédite, moins linguistique que spatiale, qui est créée par l'érosion temporelle des liens internet.

\subsection{Insertion dans l'écosystème internet}

Pour créer des bannières, certains utilisent des services d'autres sites (MySpace, ou Image Shack, un site d'hébergement de photographies), qui sont promus sur la bannière même (en bas à gauche et à droite, respectivement), et assortis d'un hyperlien vers My Banner Maker ${ }^{8}$. Ces bannières (fig. 6) constituent une autre façon de styliser profil et identité : le pseudonyme est mis en exergue sous le nom du gang, les Hoover du Westside, ou 107, que l'on retrouve aussi dans le pseudonyme de Hoova Princess.

Fig. 6. Bannière de Hoova Princess

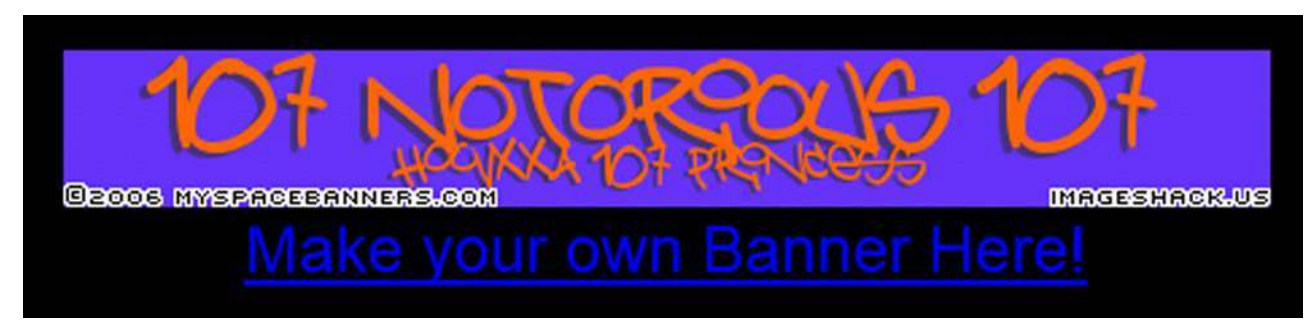

Le choix des couleurs de cette bannière est sémiotiquement pertinent : l'utilisatrice rend hommage aux Crips, alliés des Hoover, dans le choix de son fond bleu, et la couleur orange qu'elle utilise pour écrire le texte est l'emblème de son gang. Elle n'est pas responsable de la présence des références aux sites sur la bannière, éléments obligatoires prouvant leur utilisation pour composer la bannière.

17 La péremption des liens ou des images extérieurs est symbolisée par des pictogrammes comme (croix rouge sur fond blanc ou symbole du lien cassé), et témoigne de la temporalité de la page personnelle (fig. 7). Ici, l'image est remplacée par son URL d'origine, et il ne reste que les ancres HTML pour témoigner que les liens existaient au moment de la conception du profil.

Fig. 7. Liens cassés (Baby Loc)

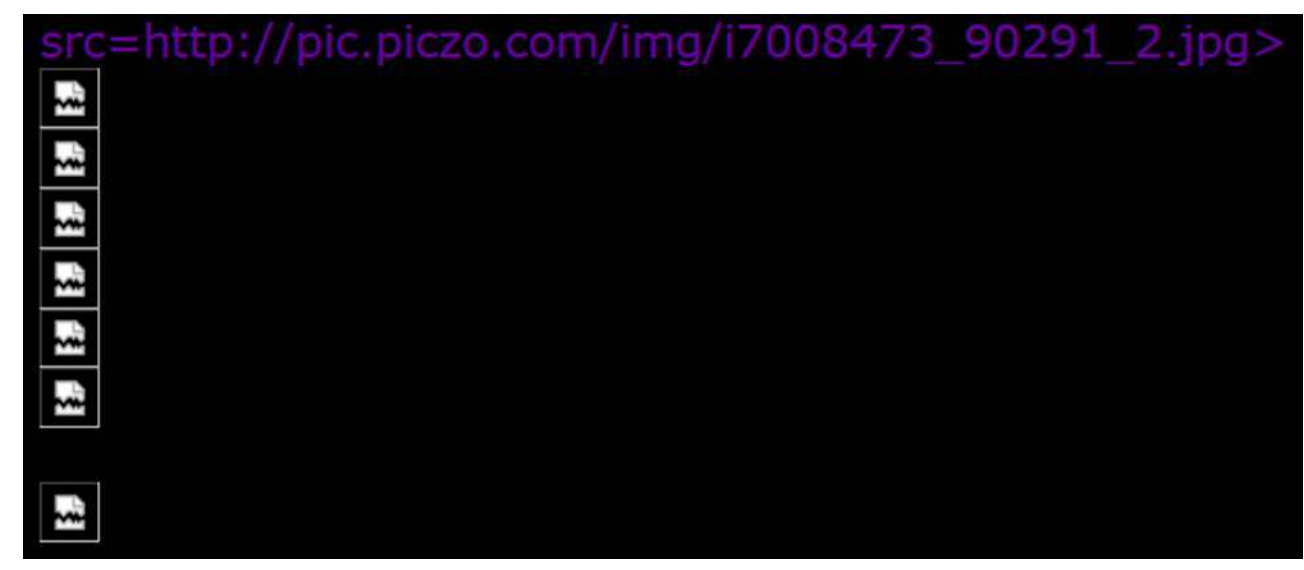

Itinéraires, 2014-1 | 2015 

[adresse sur MySpace] » («pour les femmes, pour voir quelques nouvelles photos sexy [... ], allez à [adresse] ») (Payday King). Le membre invite les utilisatrices à se rendre sur son autre profil personnel, dans une plateforme différente, qui ne joue donc pas le même rôle : son identité est scindée entre celle de BP et celle (séductrice) de l'autre site. Cela inscrit la page personnelle dans un écosystème complexe et montre le soin apporté à tous les réseaux fréquentés par l'utilisateur: son portrait est fragmenté en plusieurs lieux, d'où le pont vers un autre site, et il considère que les autres pratiquent la même fragmentation. plusieurs types de contenu, tout en le plaçant dans un contexte plus large, tiré de leur vie en ligne. Le profil est l'endroit de l'entassement (parfois illisible) d'informations qui créent l'identité en ligne. La page personnelle est stylisable à merci, et ceci permet une accumulation d'éléments-signature de soi et de son gang.

\section{Sémiotique de la page personnelle}

Les éléments des pages personnelles se superposent horizontalement ou verticalement, respectivement soit hors de la page elle-même avec des liens hypertextes en et hors site, soit dans la page, avec les diverses sections du profil, représentées par des sauts de ligne ou des illustrations qui coupent les zones de texte.

21 Les gangs sont des organisations où les membres multiplient les éléments sémiotiques identitaires (Richardson 2006). Plusieurs strates cryptiques sont mobilisées: choix du placement du bandana dans les poches du pantalon indiquant l'appartenance à un gang, messages paraverbaux comme les signes mimés de la main depuis les années 1950 (Kontos et Brotherton 2007), fig. 8.

Fig. 8. Signe d'appartenance aux Crips (D-Tay the Gunner)

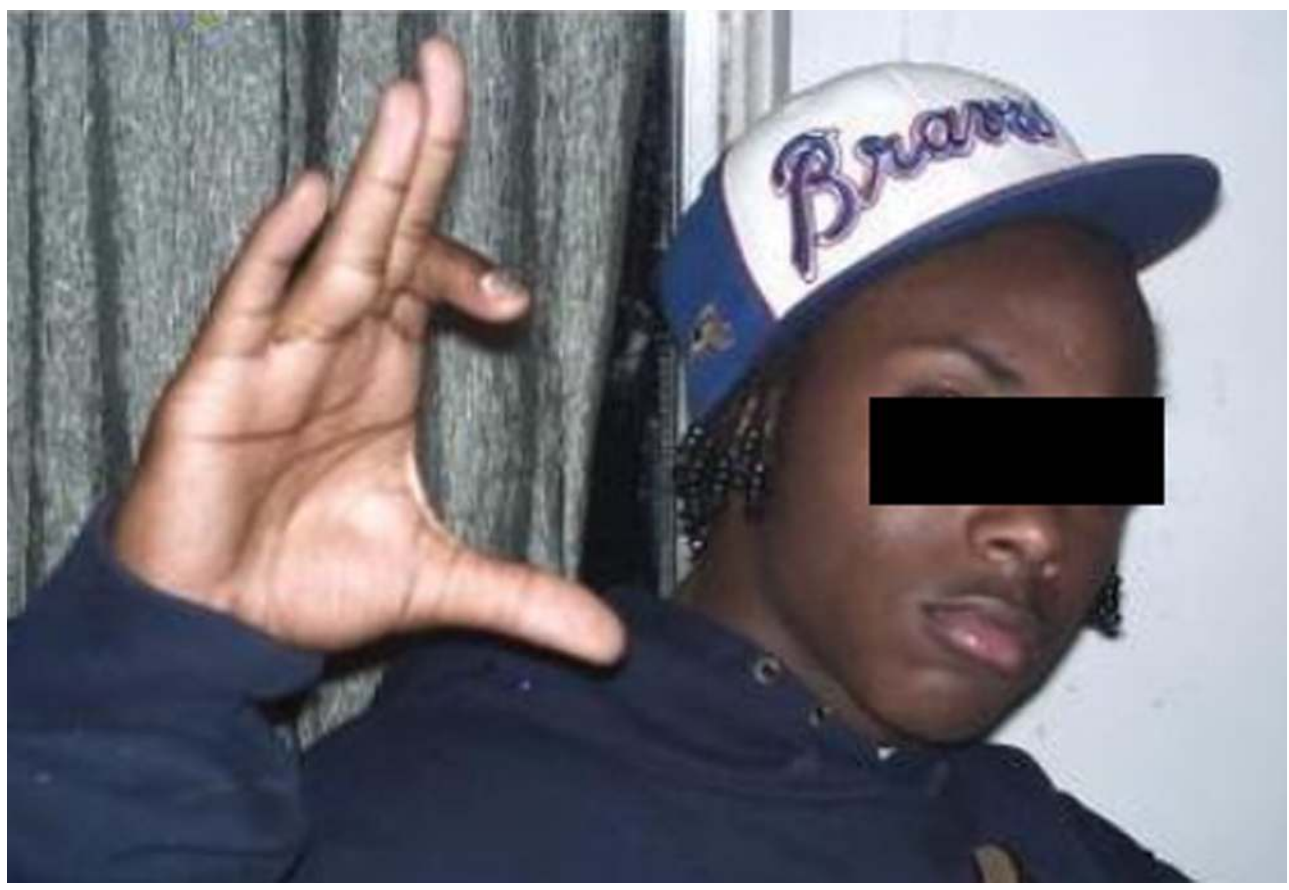

Itinéraires, 2014-1 | 2015 
Un groupe d'appartenance, d'autant plus lorsqu'il est illégal et minoritaire par rapport à la société dans laquelle il évolue, établit toujours des codes, qui permettent de créer une identité de groupe tout en mettant les non-membres à l'écart, en l'occurrence, le gang ennemi dans la fig. 9.

Fig. 9. Bandana du gang ennemi en feu (Big Herc)

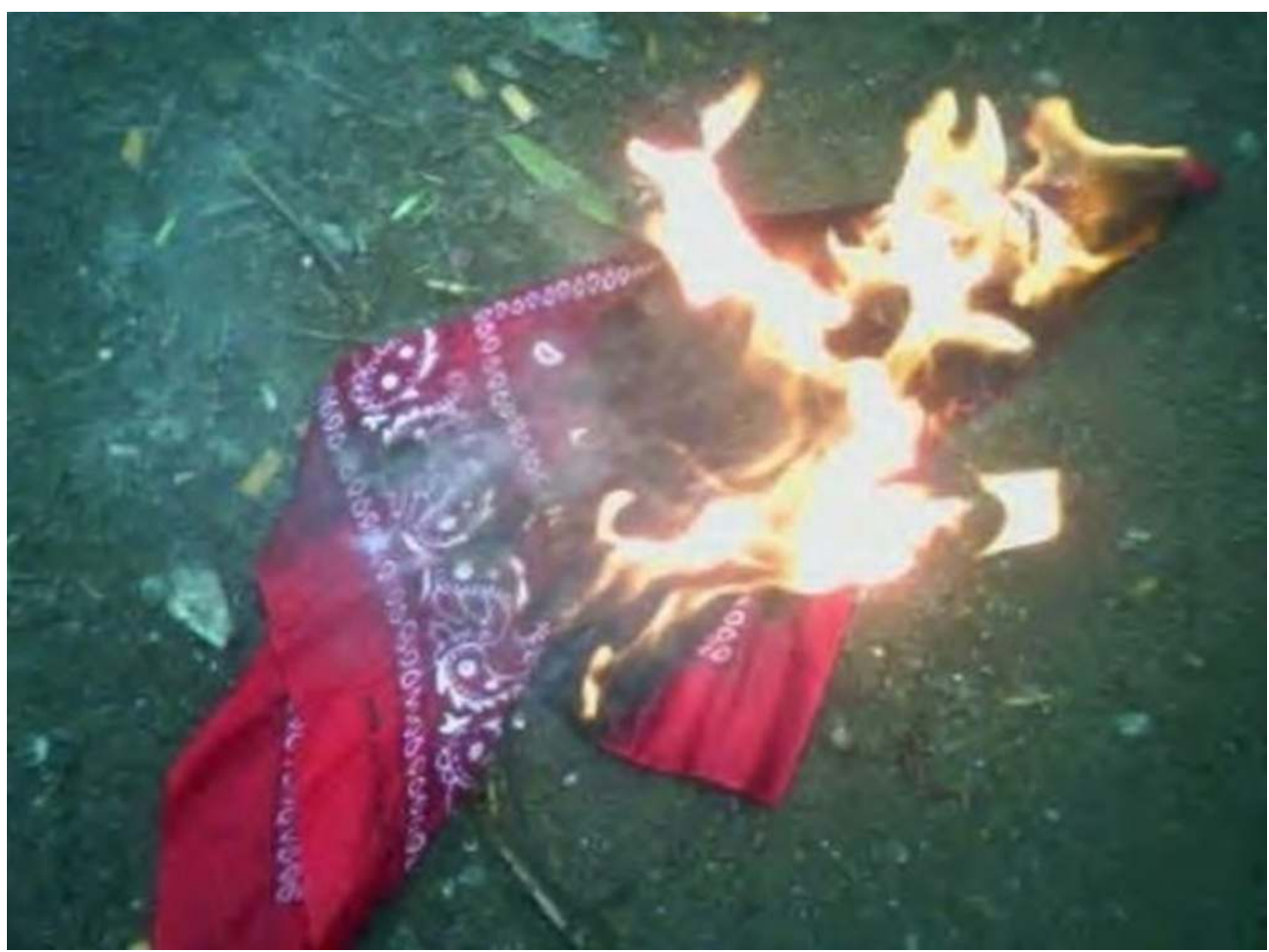

Tous les éléments de ces pages participent à une stylisation esthétisante, conforme aux attentes d'un membre de gang sur sa propre présentation (qui doit obéir aux codes qu'il respecte et grâce auxquels il s'est créé une identité).

\subsection{Stylisation du contenu}

Le choix de couleurs, d'illustrations, de polices différentes montre un soin particulier apporté à la stylisation du profil (un seul n'a aucune stylisation chromatique), d'autant plus étonnant que certains de ces membres ne vont pas participer aux discussions sur BP.

Fig. 10. En-tête de rubrique (Bompton Treetop)

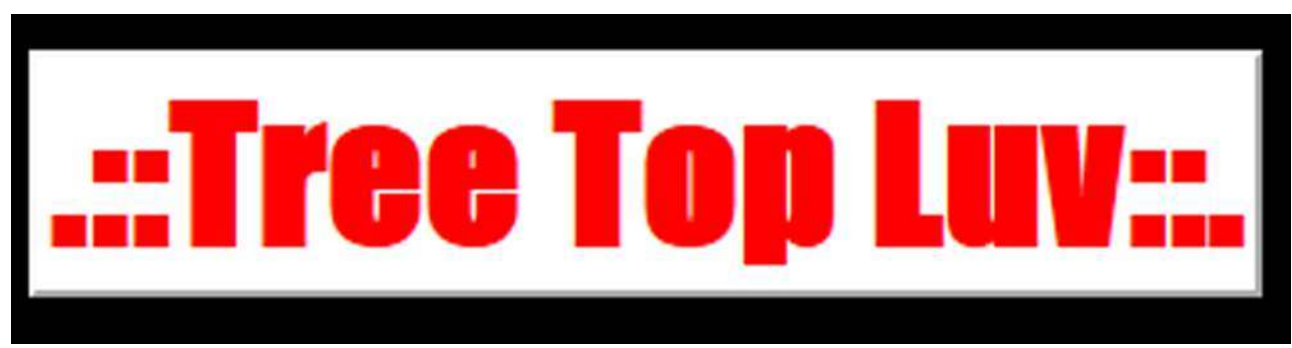

Bompton Treetop délimite les zones de texte avec des bannières créées sur d'autres sites, et qui ne sont pas en rupture avec son identité sur BP, car l'image fédère l'identité grâce 
au pseudonyme unique. Le texte est esthétisé par sa transformation en image, le choix conscient d'une police et l'adjonction de signes de ponctuation comme cadres du discours.

Dans ces textes esthétisés, la sémiotique du gang est respectée: un Blood choisit une police rouge, un Crip écrit en bleu, ou, au moins, fait apparaitre une marque péri-verbale de son appartenance à un gang (comme "Thug Life », "vie de voyou» tatouée sur le ventre de Tupac Shakur, expression-clé utilisée pour définir leur style de vie).

Elle est reprise dans une police gothique (fig. 11), insérée dans une bannière pour appuyer son identité. Cette police gothique renforce l'aspect sérieux et presque solennel du contenu; l'utilisateur appuie la dénotation par la connotation. Cette police rivalise avec les polices à effet «tag » dans les profils personnels.

Fig. 11. "Thug Life », image et mise en forme d'appartenance à un gang

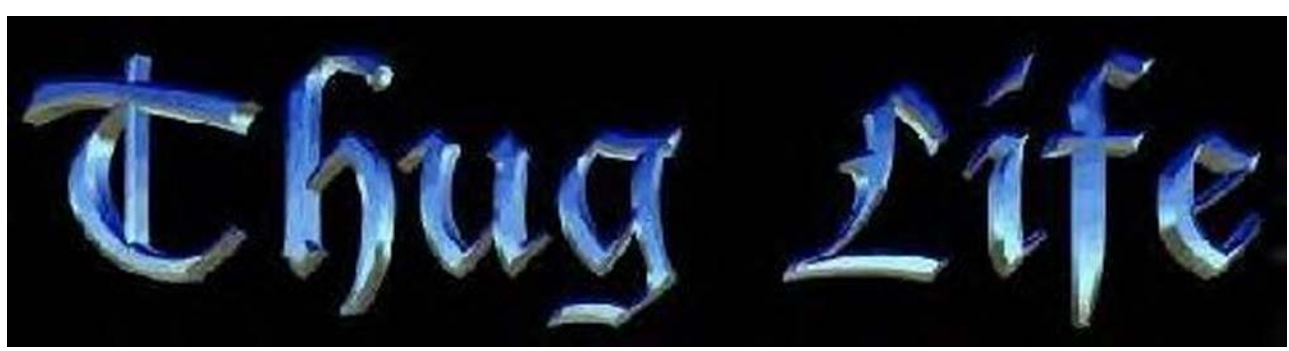

\subsection{Jeux graphiques}

Les échanges et contenus propres aux membres de gang respectent plusieurs règles, dont des graphies alternatives qui renforcent le sens des mots et donnent paradoxalement plus de force aux structures graphiques (Goudet 2013). Tacitement, il est tabou d'évoquer l'initiale du gang rival, et cela donne lieu à des lipogrammes ou des substitutions graphiques.

Les tactiques pour contourner le symbole du gang ennemi sont diverses : soit il est évité, soit il est remplacé, soit il est neutralisé : le $<\mathrm{C}>$ ou le $<\mathrm{B}>$ peuvent être suivis par $<\mathrm{K}>$ pour symboliser le mot killer et ainsi porter atteinte au gang ennemi. Même en position initiale d'une phrase, ces lettres ne portent jamais de majuscule. À l'inverse, les Crips doivent neutraliser la suite graphique $<\mathrm{ck}>$ (initiales de crip killer) en $<c c>$ (deux références aux Crips).

Les tactiques visuelles pour neutraliser les propos concernant les membres du gang rival varient suivant le scripteur. Delamo Block barre les lettres de son message envers les Bloods : «ALLSLOBZ\&ALLDIE » (« tous les [Bloods] meurent tous »). Des caractères extraalphabétiques sont employés pour porter atteinte aux rivaux, mais ne sont pas pris en charge par BP : Redrum Thing tente d'employer le $\langle \$\rangle$, remplacé par le pictogramme de caractère inconnu, $\langle$. PraBs lay $n$ a Dit $h$ «[Crips] étalés dans un fossé ». Il est néanmoins utilisé sur d'autres plateformes, et près de $20 \%$ des Bloods ont recours au remplacement de $<c>$ par l'avatar de $<\$>$ dans leur profil.

31 Les insultes rituelles («Slobs» pour les Bloods et «Crabs» pour les Crips) contiennent les caractères tabous pour chaque gang qui les utilise : ils contiennent parfois le $<\mathrm{k}>\mathrm{d}$ 'insulte $(c k r a b$, slobks). Les insultes sont cycliques et s'ajoutent les unes aux autres. La façon de 
dresser le portrait de l'autre, en le dévalorisant, fait partie en creux de l'identité que l'on s'attribue.

\subsection{Propriétés génériques des profils} comme «you », « $u$ ». Une identification discrète (non signalée par des éléments linguistiques grammaticaux) du coénonciateur est aussi possible et, dans cette analyse pragmatique, j'étudie deux aspects de l'interaction avec un potentiel lecteur : le partage didactique de connaissances et la séduction, qui passe par des écrits répondant au style de la petite annonce. 


\subsection{Aspect didactique des profils}

39 Les profils étudiés comportent parfois une partie didactique, ou explication de codes cryptographiques pour les autres internautes. Le sens est exprimé plusieurs fois : pour les connaisseurs, qui n'ont pas besoin de l'explication, et pour les profanes, qui apprennent ainsi les codes afférents au gang. La redondance des marques peut être l'indice que ce n'est pas un code utilisé au quotidien, et que cette didactique s'adresse autant au scripteur (ou recopieur) qu'aux lecteurs.

Fig. 12. Didactique du profil (Hoova Princess)

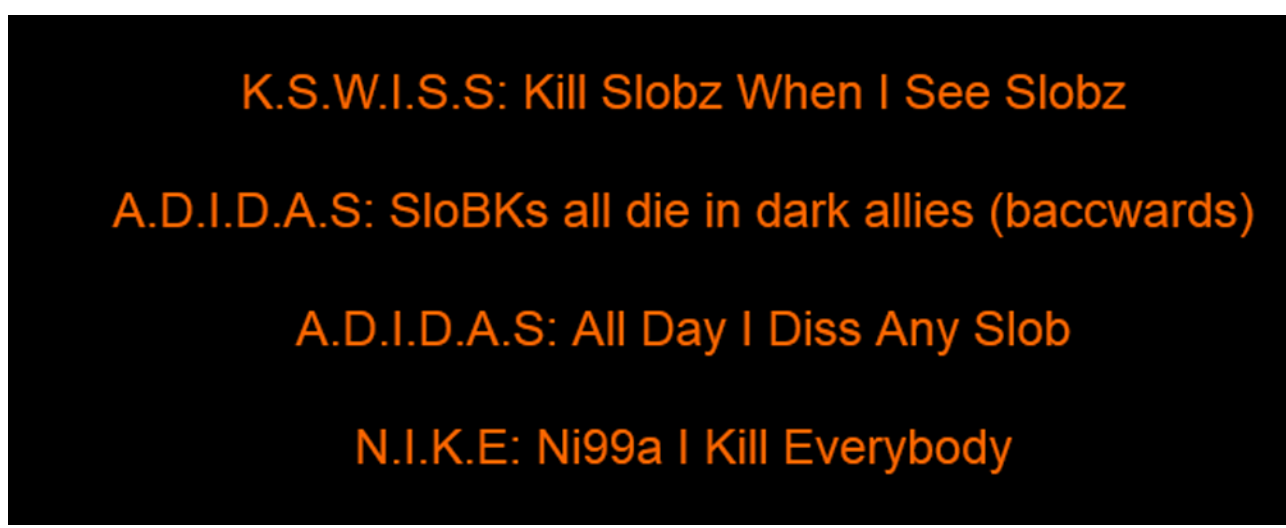

Les initiales de ce lexique sont thématiques : elles sont des reprises de marques connues, familières aux membres de gangs. Leur détournement au profit d'une nouvelle signification est un phénomène connu de récupération et de développement de stratégies de langage secret ou codé.

41 Adidas offre une lecture anacyclique, avec un sens différent : soit « All Day I Diss Any Slob " («Toute la Journée, Je Dénigre N’importe quel [Blood]»), soit «Slo[b]s All Die In Dark All [ey]s» («Les [Bloods] Meurent Tous dans des Allées Sombres»): seul compte le détournement sémantique, au détriment du message que l'on fait passer, sa seule contrainte étant le fait qu'il soit injurieux envers l'autre gang, ou violent, comme « Ni[gg]a I kill everybody ( (« Négro, Je Tue Tout le monde »).

Le code couleurs reprend celui des Hoover et permet l'identification du gang sans même avoir lu le texte. Celui-ci reprend des codes sémiotiques de l'opposition Blood/Crip. Lexicalement, les mots employés font partie soit de l'argot général des gangs, comme " diss " (« dénigrer »), soit des insultes centrées sur le gang comme «slob» (les «bons à rien » pour les Bloods).

Les parties didactiques peuvent être écrites sous formes de courts poèmes, car l'eurythmie aide à la mémorisation :

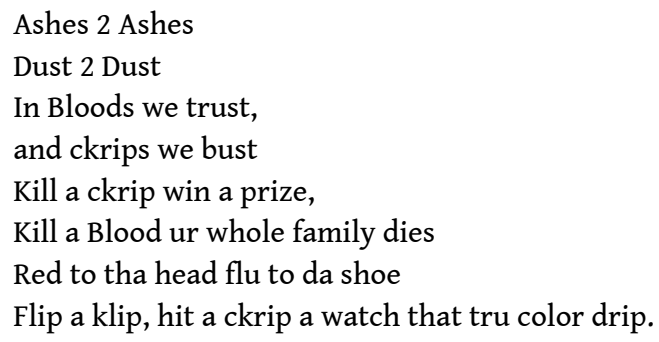


Poussière, tu es poussière

et tu retourneras à la poussière

On croit aux Blood

On tue les Crips

Tue un Crip, gagne un lot

Tue un Blood, toute ta famille meurt

Rouge jusqu'à la tête, Bleu à nos pieds

Mets un chargeur, tire dans un Crip et regarde la vraie couleur couler. des premières informations demandées, avant l'appartenance ethnique ou la profession. Trente-cinq pour cent des membres utilisent leur espace personnel comme lieu pour placer une petite annonce matrimoniale. Un exemple de ces adresses de séduction est présenté dans le profil de Hood Banga, qui expose ce qu'il recherche, puis dresse son propre portrait : 
Fig. 13. Recherche amoureuse (Hood Banga)

And what I like in a Girl is a girl who is thick (not fat) a Girl who got a $\$ \$$ you know what I mean (got a body like damn!) call me if u need some loving for tonite (402) 453-0144. On tha real don't call if $u$ on that bullsh**t on bloods I will hang up on yo stupid a\$\$.

\section{About Lonzo From FLATLAND 16th u kno tha BOTTOMS.... \\ *'m about 5`11 \\ *I'm dark skin \\ *I got a sexy a\$\$ body \\ *I got dark eyes \\ u all ready know i goy to stay fresh never dusty I'm always on ma pezzz and quzzz}

Et ce que j'aime chez une Fille c'est une fille qui est enrobée (pas grosse) une fille qui a des fe\$\$es tu vois ce que je veux dire (qui a un corps genre wahou!) appelezmoi si vous voulez de l'amour ce soir [Numéro de téléphone]. C'est pour de vrai ne m'appelle pas si tu dis des conneries sur les Bloods, je te raccrocherai à la gueule connard.

Cette petite annonce se double d'un avertissement : pour joindre le membre hors ligne il faut avoir une raison valable et ne pas manquer de respect aux Bloods, ou il refusera le dialogue. Donner son numéro de téléphone, coordonnée hors ligne, dépasse le cadre de l'identité numérique: le préfixe téléphonique américain donne une indication géographique à l'échelle de la ville, et dévoile donc des coordonnées physiques de l'internaute, ce qui est généralement très déconseillé ${ }^{11}$. Hood Banga multiplie ainsi les façons de le contacter, à partir de sa page personnelle, et rompt le mur qui oppose hors et en ligne.

Cette recherche de partenaire n'est pas exempte de marques d'appartenance à un gang, et la fig. 14 montre que l'appartenance au gang peut être déterminante dans le choix d'un partenaire : elle ne veut pas de « ho ass rabs » (littéralement, des « [Crips] à cul de pute »). 
Fig. 14. Recherche amoureuse (Piru Queen)

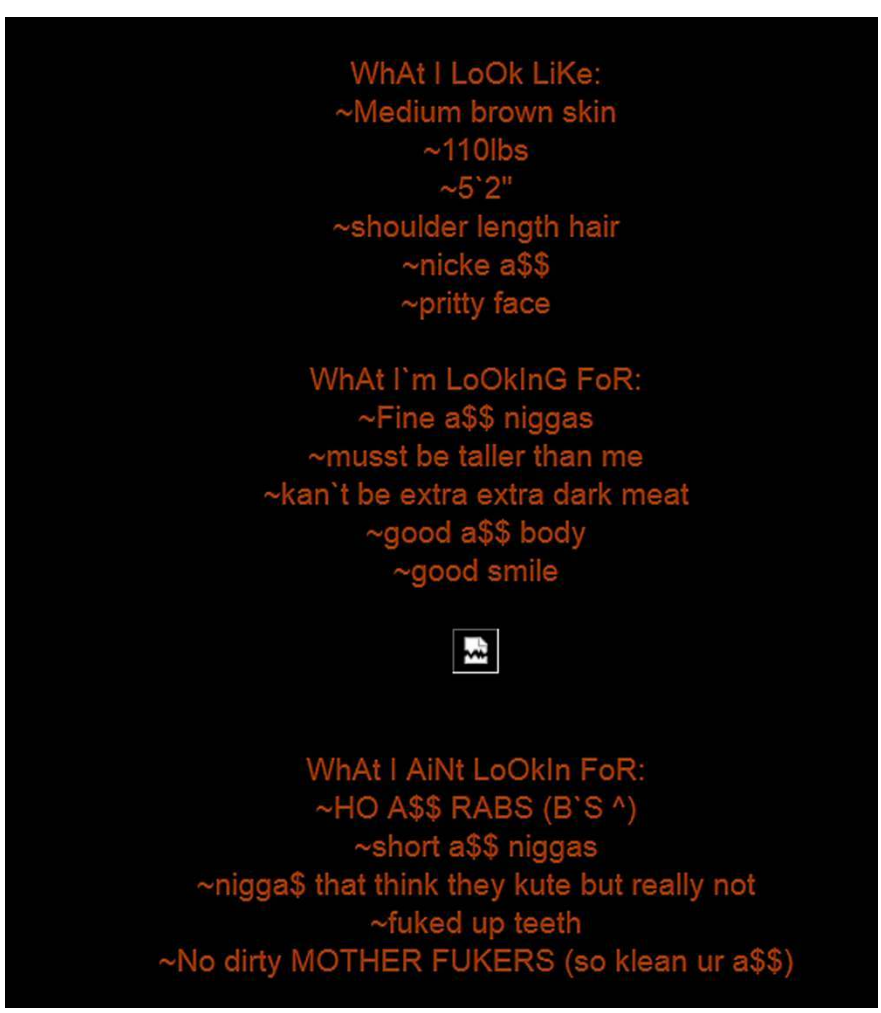

Piru Queen, comme Hoova Princess, structure sa demande suivant le plan suivant: d'abord, une salutation, une description personnelle, puis ce qui l'attire et la repousse. La petite annonce est le premier élément tourné vers l'autre, donc ce qui lui tient le plus à cœur. Il est suivi d'un shoutout. Elle va donc du moins connu (potentiels partenaires ou candidats) au plus familier, écrit pour elle-même, qui connaît déjà ceux qu'elle cite.

Ces petites annonces sont rédigées sous forme de listes, faciles à lire et à composer. Les répulsions et les attirances sont écrites pour de potentiels destinataires, mais créent paradoxalement un portrait de leurs auteurs.

\section{Conclusion}

Le profil est un lieu de l'entre-deux, entre la participation à une communauté, et à une vitrine de soi, ou du monde dans lequel le participant évolue hors ligne. Des codes sémiotiques divers s'affrontent ou se complètent, et le connotatif est privilégié. Un profil d'utilisateur est toujours à lire en rapport avec son contexte plus général (le forum ou le site sur lequel il a été créé), mais aussi par rapport au type de communauté qui fédère ces utilisateurs. Le réseau de sociabilité passe par l'hors et l'en ligne. On y retrouve des caractéristiques du journal en ligne, des coordonnées pouvant permettre l'identification hors ligne (courriel, numéro de téléphone).

La stylistique imposée aux membres de gangs leur apporte une certaine crédibilité, valeur importante, dans un milieu où l'e-thug ${ }^{12}$ est méprisé et où les gangs tentent de s'imposer en ligne (Esperanza 2010). Certaines caractéristiques formelles servent à asseoir le membre et son écosystème propre dans la dynamique du gang, en utilisant les outils typographiques à disposition. 
Malgré l'agressivité et la violence envers le gang rival, thèmes communs de ces profils, les membres interagissent didactiquement avec les autres, en copiant des phrases rituelles ou des lexiques dans leurs présentations, tout en essayant de trouver un partenaire. Le profil est le lieu de communication entre le très intime, voire le hors ligne, et l'agonalité envers les membres de l'autre gang, systémique dans la création identitaire du membre de gang afro-américain.

\section{BIBLIOGRAPHIE}

Atoon, Patrick « Tricky », 1992, «Crenshaw Mafia Gang Bloods », Rap Dictionary, [En ligne], http://www.rapdict.org/Crenshaw_Mafia_Gang_Bloods, consulté le 13 janvier 2015.

Coutant, Alexandre et Stenger, Thomas, 2014, Identités Numériques, Paris, L’Harmattan.

Deseilligny, Oriane, 2012, « La mémoire appareillée : dispositifs numériques et écriture de soi », ESSACHESS - Journal for Communication Studies, vol. 5, nº 2 (10), p. 95-105.

Esperanza, 2010, Gangs 101-Understanding the Culture of Youth Violence, Philadelphie, Esperanza.

Georges, Fanny, 2008, «L'identité numérique dans le web 2.0 », Le mensuel de l'Université, n²7, [En ligne], http://fannygeorges.free.fr/doc/georgesf_mensueluniversite.pdf.

Goudet, Laura G., 2013, « Alternative Spelling and Censorship: The Treatment of Profanities in Virtual Communities », dans Denis Jamet et Manuel Jobert (dir.), Aspects of Linguistic Impoliteness, Newcastle upon Tyne, Cambridge Scholars Publishing.

Kontos, Louis et Brotherton, David, 2007, Encyclopedia of Gangs, Westport, Greenwood Press.

Paveau, Marie-Anne, 2012, « Réalité et discursivité. D’autres dimensions pour la théorie du discours », Semen, $\mathrm{n}^{\circ}$ 34, p. 95-115.

Pullum, Geoffrey, 2004, « Snowclones: lexicographical dating to the second », Language Log, [En ligne], mis en ligne le 28 janvier 2004, http://itre.cis.upenn.edu/ myl/languagelog/ archives/000350.html, consulté le 13 janvier 2015.

Richardson, Elaine B., 2006, Hiphop Literacies, New York, Routledge.

Smitherman, Geneva, 2000, Black Talk: Words and Phrases from the Hood to the Amen Corner, Boston, Houghton Mifflin.

Urban Dictionary, [1999] 2006, www.urbandictionary.com\adidas, consulté le 13 janvier 2015.

Wenger, Etienne, 1998, Communities of Practice: Learning, Meaning, and Identity, Cambridge, Cambridge University Press.

Windley, Phillip J., 2005, Digital Identity, Sebastopol, O’Reilly.

\section{NOTES}

1. www.blackplanet.com. 
2. Le rapport entre Bloods et Crips est bien respecté : il y aurait 30000 Crips, contre 15000 à 20000 Bloods (Kontos et Brotherton 2007).

3. Aucune autorisation ne leur a été demandée, étant donné que certains de ces membres ne sont plus présents sur BP. En revanche, leur profil est accessible sans compte sur BP, mais les liens sont maquillés par Bitly.

4. Le .gif (Graphic Interchangeable Format), est traditionnellement employé en ligne pour des images animées, alors que le .jpg ou .jpeg (Joint Photographic Experts Group) est le format le plus utilisé pour transmettre des images en ligne.

5. The Bottoms est une zone près d'Inglewood, Californie, majoritairement occupée par des Bloods (Atoon 1992).

6. L'hyperlien semble renvoyer à sa page (http://bit.ly/1qEiu3y), mais en réalité, il pointe vers l'aide de BP, section «Liste d'amis et favoris », (http://bit.ly/1oH1aPG, dernière consultation le 7 mai 2013).

7. 2Pac ou Tupac Shakur, artiste de hip hop à la carrière fulgurante.

8. http://www.mybannermaker.com/. Dernière consultation le 19 avril 2013.

9. L'Urban Dictionary (1999) recense une version plus agressive envers les Bloods : « At Dawn/Dusk I Dust All Slobs ».

10. Trope internet courant, où un patron textuel est transformé en cliché discursif qui sert à la création de variantes inédites, souvent par substitutions paradigmatiques.

11. C'est le cas des pages dédiées à la sécurité sur les réseaux sociaux, comme http:// www.wikihow.com/Be-Safe-on-the-Internet et http://bit.ly/1sx6AfH (dernier accès : 10 avril 2014).

12. Personne se faisant passer pour un membre de gang sur Internet.

\section{RÉSUMÉS}

Cet article est une étude de cinquante-cinq pages de profils de membres autoproclamés de gangs (Bloods et Crips) sur Black Planet (BP), le plus important réseau social afro-américain. Ces profils partagent des caractéristiques communes, comme la représentation de son propre gang (" repping»), et l'insulte du gang rival («dissing»). L'examen de la projection de leur page dans un écosystème plus important (en- et hors ligne, sur BP et d'autres plateformes) et une analyse sémiotique des éléments (choix chromatiques et typographiques, etc.) permet de dégager la "stylisation numérique » de ces monologues ainsi que la jonction entre discours numérique et rhétorique de gang, dans l'espace ouvert mais intime du profil personnel.

This article is a study of fifty-five profile pages of self-proclaimed gang members (Bloods and Crips) on Black Planet (BP), the biggest African American website. These share formal characteristics, as "repping" "representing" one's gang, or "dissing" the rival gang. Considering the projection of their pages in a wider ecology (on- and off-line, on and off BP) and analyzing semiotic elements (chromatic, typographic choices, etc.), allows to highlight the "digital stylization" of these monologues and the junction between computer-mediated discourse and gang rhetoric, in the intimate yet open space of the profile. 
INDEX

Mots-clés : gangs, vernaculaire afro-américain, monologue, pragmatique, littératie

Keywords : African American language, pragmatics, literacy

\section{AUTEUR}

LAURA GABRIELLE GOUDET

Université Paris 13, Sorbonne Paris Cité, Pléiade (EA 7338) 